\title{
Risk factors for aspiration pneumonia after definitive chemoradiotherapy or bio- radiotherapy for locally advanced head and neck cancer: a monocentric case control study
}

Sadayuki Kawai ${ }^{1}$, Tomoya Yokota ${ }^{1 *}$, Yusuke Onozawa ${ }^{2}$, Satoshi Hamauchi ${ }^{1}$, Akira Fukutomi ${ }^{1}$, Hirofumi Ogawa ${ }^{3}$, Tsuyoshi Onoe ${ }^{3}$, Tetsuro Onitsuka ${ }^{4}$, Takashi Yurikusa ${ }^{5}$, Akiko Todaka', Takahiro Tsushima' ${ }^{1}$, Yukio Yoshida', Yosuke Kito ${ }^{1}$, Keita Mori ${ }^{6}$ and Hirofumi Yasui ${ }^{1}$

\begin{abstract}
Background: Chemoradiotherapy (CRT) and bio-radiotherapy (BRT) are recognized as standard therapies for head and neck cancer (HNC). Aspiration pneumonia after CRT or BRT is a common late adverse event. Our aim in this study was to evaluate the cause-specific incidence of aspiration pneumonia after CRT or BRT and to identify its clinical risk factors.
\end{abstract}

Methods: We performed a retrospective analysis of 305 patients with locally advanced HNC treated by CRT or BRT between August 2006 and April 2015.

Results: Of these 305 patients, 65 (21.3\%) developed aspiration pneumonia after treatment. The median onset was 161 days after treatment. The two-year cause-specific cumulative incidence by CRT or BRT was 21.0\%. Multivariate analysis revealed five independent risk factors for aspiration pneumonia, namely, habitual alcoholic consumption, use of sleeping pills at the end of treatment, poor oral hygiene, hypoalbuminemia before treatment, and the coexistence of other malignancies. A predictive model using these risk factors and treatment efficacy was constructed, dividing patients into low- (0-2 predictive factors), moderate- (3-4 factors), and high-risk groups (5-6 factors), the two-year cumulative incidences of aspiration pneumonia of which were 3.0, 41.6, and 77.3\%, respectively. Aspiration pneumonia tended to be associated with increased risk of death, although this was not statistically significant (multivariate-adjusted hazard ratio $1.39, P=0.18$ ).

Conclusion: The cause-specific incidence and clinical risk factors for aspiration pneumonia after definitive CRT or BRT were investigated in patients with locally advanced HNC. Our predictive model may be useful for identifying patients at high risk for aspiration pneumonia.

Keywords: Head and neck cancer, Aspiration pneumonia, Risk factor, Chemoradiotherapy, Case-control study

\footnotetext{
* Correspondence: t.yokota@scchr.jp

${ }^{1}$ Division of Gastrointestinal Oncology, Shizuoka Cancer Center, 1007

Shimonagakubo, Nagaizumi, Sunto-gun, Shizuoka 411-8777, Japan

Full list of author information is available at the end of the article
} 


\section{Background}

Chemoradiotherapy (CRT) is a standard treatment for locally advanced head and neck cancer (HNC) [1]. Radiotherapy (RT) with cetuximab, defined as bio-radiotherapy (BRT), is also considered as a treatment option for patients with locally advanced HNC [2]. Compared with radical surgery, CRT and BRT have an advantage of preserving organ function and patients' quality of life; however, their toxicities are not less harmful than the risks associated with surgery. In the previous clinical trial RTOG 91-11 [3], noncancer-related death was more common among patients treated with CRT than with RT alone in a further followup, despite the higher rates of laryngeal preservation [4]. This suggests that patients cured by CRT need appropriate management against late toxicity.

Aspiration pneumonia is recognized as pneumonia secondary to the inhalation of food particles, saliva, or gastric acid. Patients with HNC who have undergone definitive CRT tend to have swallowing dysfunction due to mucositis during the treatment period or due to radiation-induced fibrosis of the oropharyngeal musculature after completion of the treatment [5]. Szczesniak et al. [6]. reported that approximately $52 \%$ of patients who received RT and 69\% who received CRT suffered from dysphasia after treatment, and aspiration pneumonia accounted for $19 \%$ of non-cancer-related deaths. Additionally, $\mathrm{Xu}$ et al. [7]. suggested that aspiration pneumonia was a poor prognostic factor for patients with HNC who received CRT. Therefore, clinicians should assess the risk of aspiration pneumonia in order to identify patients for whom efforts to prevent it should be implemented.

The purpose of this study was to identify clinical risk factors for aspiration pneumonia after definitive CRT or BRT for patients with advanced HNC. In particular, we focused on the cause-specific incidence of aspiration pneumonia, taking competing events of death and resection of the primary lesion into account.

\section{Methods \\ Study population}

Three hundred and forty patients with HNC who received definitive concurrent CRT or BRT at Shizuoka Cancer Center between August 2006 and April 2015 were identified from medical records. Of these, 35 patients with a recurrent or metastatic lesion or resection of the primary lesion before CRT were excluded. Patients with other malignancies were included only if HNC was considered to be the factor most strongly determining their prognosis. Finally, 305 patients were included in this analysis. This study was approved by the Institutional Review Committee of Shizuoka Cancer Center (Shizuoka, Japan) and met the standards set forth in the Declaration of Helsinki.

\section{Study covariates}

We retrospectively collected data on the occurrence of aspiration pneumonia, time to onset of aspiration pneumonia, and overall survival (OS) from the end of treatment. Background covariate candidates for factors predictive of aspiration pneumonia included the following: tumor site, age, gender, Eastern Cooperative Oncology Group (ECOG) performance status, body mass index, TNM staging according to the AJCC/UICC TNM classification, tumor histology, smoking status, habitual alcoholic consumption, distance between the patients' home and the hospital, family members in the same household, use of proton pump inhibitors (PPIs) or $\mathrm{H}_{2}$ blockers, use of angiotensin-converting enzyme (ACE) inhibitors or angiotensin II receptor blockers (ARBs), use of sleeping pills and main feeding at the end of the treatment, presence of gastrostomy during the treatment, oral hygiene, serum albumin (ALB) and hemoglobin $\mathrm{Hb})$ levels before treatment, coexistence of other malignancies before treatment, and Charlson comorbidity index. We defined habitual alcoholic consumption as the drinking of alcohol four or more days a week, and poor oral hygiene as the presence of moderate or more severe dental plaque assessed by a dentist and/or a dental hygienist. Charlson comorbidity index is a tool for predicting mortality by classifying or weighting comorbidities [8].

We also collected the following treatment-related covariate data: presence or absence of induction chemotherapy, chemotherapy regimen, irradiation technique [conventional three-dimensional conformal radiation therapy (3DCRT) or intensity-modulated radiation therapy (IMRT)], irradiation field, treatment efficacy evaluated according to Response Evaluation Criteria in Solid Tumors ver. 1.1 [complete response (CR) or non-CR], mucositis and dysphagia during treatment evaluated by Common Terminology Criteria for Adverse Events ver. 4.0, and decreases of $\mathrm{ALB}, \mathrm{Hb}$, and body weight after treatment.

\section{Aspiration pneumonia}

Because it is sometimes difficult to clearly distinguish aspiration pneumonia from other types of pneumonia, different definitions of aspiration pneumonia were used in previous studies [9-11]. Therefore, in this study, we defined aspiration pneumonia as a clinical condition that met all of the following criteria: (i) Patients had both subjective and objective symptoms suggesting pneumonia. Subjective symptoms included wet cough, sputum, and fever. Objective symptoms included the presence of coarse crackles in the chest, elevated inflammatory markers (e.g. white blood cell count or C-reactive protein), or image findings (e.g. infiltration on a chest X-ray or consolidation in chest computed tomography). (ii) The presence of aspiration was suspected clinically (choking or delayed swallowing) or by endoscopic or video-fluorographic examinations. (iii) No evidence of 
micro-organisms that cause atypical pneumonia, such as Legionella and Mycoplasma.

\section{Statistical analysis}

The cause-specific cumulative incidence of aspiration pneumonia was estimated with nonparametric cumulative incidence functions, taking competing events of death and resection of the primary lesion into account. To investigate potential risk factors for aspiration pneumonia, univariate analysis was carried out for all covariates using Fisher's exact test, and covariates showing statistical significance were further analyzed using a multivariate logistic regression model. To construct a predictive model, we automatically selected covariates extracted from univariate analysis, and compared the goodness-of-fit among many models on the basis of the stepwise Akaike information criterion (AIC) method [12]. The minimum value from the AIC procedure allows us to select appropriate predictive factors to construct an optimal predictive model objectively. The concordance index to evaluate the discriminatory ability of the model was calculated using the final regression model [13].

The OS time was calculated from the date of treatment end to the date of death due to any cause or to the last date of confirmed survival. Survival rates were estimated using the Kaplan-Meier method. To estimate the association of covariates with overall survival, univariate analysis was carried out using the log-rank test. All statistically significant covariates in univariate analysis were analyzed in multivariate analysis using the Cox regression model.

All statistical tests were two-sided, and $P \leq 0.05$ was considered significant. Statistical analyses were performed using EZR software (Saitama Medical Center, Jichi Medical University, Saitama, Japan) [14].

\section{Results}

Among the 305 patients, 65 (21.3\%) developed aspiration pneumonia after CRT or BRT. Patients' baseline and treatment-related characteristics are summarized in Table 1 . The median age of the patients was 65 years (range 19-83) and $95.1 \%$ of them had ECOG PS of 0 to 1. Cisplatin, carboplatin, and cetuximab were concurrently used in $77.1,13.7$, and $9.2 \%$ of patients, respectively. Seventy-six (24.9\%) of the patients received induction chemotherapy, and $87.5 \%$ of them were treated with the combination of docetaxel, cisplatin, and fluorouracil. Additionally, $96.0 \%$ of all patients had received systematic oral care [15] since initiation of the treatment. Thirty-six (11.8\%) patients had coexisting malignancies included multiple primary $\mathrm{HNC}$, esophageal cancer, gastric cancer, prostate cancer, lung cancer, and renal cancer. All of these cancers were found at an early stage by routine endoscopic or computed tomography screening. After definitive CRT or BRT, 30 (9.8\%) patients underwent resection of the primary lesion and 45 (14.7\%) underwent neck dissection for a residual lesion or recurrence.

The median time from the end of treatment to aspiration pneumonia events was 161 days (range 3-1623). The median follow-up time was 892 days. The two-year cumulative incidences of aspiration pneumonia and competing events of death and resection of the primary lesion were $21.0 \%$ [95\% confidence interval (CI) 16.4$26.0 \%$ ], $12.9 \%$ (9.2-17.4\%), and 6.2\% (3.7-9.5\%), respectively (Fig. 1).

Univariate and multivariate analyses identified five independent risk factors for aspiration pneumonia, namely, habitual alcoholic consumption, poor oral hygiene, coexistence of other malignancies, hypoalbuminemia before treatment, and the use of sleeping pills at the end of treatment (Table 2). A difference in the types of sleeping pills (benzodiazepines or others) used was not associated with the onset of aspiration pneumonia (odds ratio 0.95, 95\% CI, $0.37-2.39, P=1.00)$. Of 193 patients with poor oral hygiene before treatment, 135 had been followed up by dentists three months after the treatment. In total, 87 of 135 patients in whom oral hygiene had improved three months after the treatment had a significantly lower frequency of aspiration pneumonia than 48 patients who had poor oral hygiene ( $18.3 \%$ vs. $54.1 \%, P=0.00003)$.

Next, we attempted to construct a predictive risk model of aspiration pneumonia from the results of univariate analysis. As a result of AIC stepwise selection, six predictive factors, consisting of the five risk factors extracted from the multivariate analysis and treatment efficacy (non-CR), were selected. Although treatment efficacy was not identified as a statistically significant risk factor, AIC stepwise selection revealed that it was a good predictive factor for the model. This predictive model well divided patients into low- (0-2 factors, $n=$ 180), moderate- (3-4 factors, $n=103)$, and high-risk groups $(5-6$ factors, $n=22)$ by the number of predictive factors, for which the estimated two-year cumulative incidences of aspiration pneumonia were 3.0\% (95\% CI, $1.1-6.5 \%)$, 41.6\% (31.0-51.8\%), and 77.3\% (51.4-90.5\%), respectively (Fig. 2). The concordance index was 0.797 .

Finally, we investigated the correlation between OS and the occurrence of aspiration pneumonia. Survival curves adjusted for the covariates from a Cox proportional hazard model indicated that the occurrence of aspiration pneumonia tended to be associated with the risk of death, but this was not statistically significant (hazard ratio, 1.39; 95\% CI, 0.85-2.27; $P=0.18$ ) (Fig. 3).

\section{Discussion}

The important goals of treatment in patients with HNC are not only a cure but also the preservation of quality 
Table 1 Patients' characteristics

\begin{tabular}{|c|c|c|c|}
\hline Background & $\mathrm{n}(\%)$ & Distance from the hospital & \\
\hline Age & & $<10 \mathrm{~km}$ & $92(30)$ \\
\hline$<65$ years & $149(49)$ & $\geq 10 \mathrm{~km}$ & $213(70)$ \\
\hline$\geq 65$ years & $156(51)$ & Family members in the same household & \\
\hline Gender & & Yes & $258(85)$ \\
\hline Male & $266(87)$ & No & $47(15)$ \\
\hline Female & $39(13)$ & Use of ACEi or ARB & \\
\hline ECOG performance status & & ARB & $53(17)$ \\
\hline 0 & $181(59)$ & ACEi & $2(1)$ \\
\hline 1 & $109(36)$ & No & $250(82)$ \\
\hline 2 & $12(4)$ & Use of PPI or $\mathrm{H}_{2}$ blocker & \\
\hline 3 & $3(1)$ & Yes & $163(53)$ \\
\hline Body mass index & & No & $142(47)$ \\
\hline$<18.5$ & $46(15)$ & Oral hygiene before treatment & \\
\hline $18.5-25$ & $199(65)$ & Good & $100(33)$ \\
\hline$\geq 25$ & $60(20)$ & Poor & $193(63)$ \\
\hline Primary site & & Unknown & $12(4)$ \\
\hline Larynx & $45(15)$ & Coexistence of other malignancies & \\
\hline Nasopharynx & $38(12)$ & Yes & $36(12)$ \\
\hline Hypopharynx & $112(37)$ & No & $269(88)$ \\
\hline Nasal sinus & $17(6)$ & Comorbidity index & \\
\hline Oropharynx & $79(26)$ & 0 & $233(76)$ \\
\hline Oral cavity & $14(5)$ & $\geq 1$ & $72(24)$ \\
\hline T-classification & & Serum albumin before treatment & \\
\hline 1 & $28(9)$ & Within normal limits & $259(85)$ \\
\hline 2 & $110(36)$ & Less than normal range & $46(15)$ \\
\hline 3 & $66(66)$ & Hemoglobin before treatment & \\
\hline 4 & $101(33)$ & Within normal limits & $212(70)$ \\
\hline N-classification & & Less than normal range & $93(30)$ \\
\hline 0 & $63(21)$ & Use of sleeping pills at the end of treatment & \\
\hline 1 & $39(13)$ & Yes & $210(69)$ \\
\hline $2 a$ & $3(1)$ & No & $95(31)$ \\
\hline $2 b$ & $126(41)$ & Main feeding at the end of treatment & \\
\hline 2c & $60(20)$ & Oral & $127(42)$ \\
\hline 3 & $14(5)$ & Non-oral & $178(58)$ \\
\hline Tumor histology & & Presence of gastrostomy during the treatment & \\
\hline SCC & $287(94)$ & Yes & $173(57)$ \\
\hline Others & $18(6)$ & No & $132(43)$ \\
\hline Smoking status & & Induction chemotherapy & \\
\hline Never & $36(12)$ & Yes & $76(25)$ \\
\hline Past & $200(66)$ & No & $229(75)$ \\
\hline Current & $69(23)$ & Concurrent chemotherapy regimen & \\
\hline Habitual alcoholic consumption & & CDDP-based & $235(77)$ \\
\hline Yes & $121(40)$ & CBDCA-based & $42(14)$ \\
\hline No & $184(60)$ & Cetuximab & $28(9)$ \\
\hline
\end{tabular}

Table 1 Patients' characteristics (Continued)

Distance from the hospital

$2(30)$

(85)

$47(15)$

$28(9)$ 
Table 1 Patients' characteristics (Continued)

\begin{tabular}{|c|c|}
\hline \multicolumn{2}{|l|}{ Radiation technique } \\
\hline Conventional 3D-CRT & $241(79)$ \\
\hline IMRT & $64(21)$ \\
\hline \multicolumn{2}{|l|}{ Irradiation field } \\
\hline Primary lesion alone & $40(13)$ \\
\hline Hemi neck & $19(6)$ \\
\hline Whole neck & $246(81)$ \\
\hline \multicolumn{2}{|l|}{ Treatment efficacy } \\
\hline$C R$ & $199(65)$ \\
\hline Non-CR & $106(35)$ \\
\hline \multicolumn{2}{|l|}{ Body weight loss after treatment } \\
\hline$<10 \%$ & $178(58)$ \\
\hline$\geq 10 \%$ & $127(42)$ \\
\hline \multicolumn{2}{|c|}{ Serum albumin decreasing post-treatment } \\
\hline$<20 \%$ & $114(37)$ \\
\hline$\geq 20 \%$ & $191(63)$ \\
\hline \multicolumn{2}{|c|}{ Hemoglobin decreasing post-treatment } \\
\hline$<30 \%$ & $138(45)$ \\
\hline$\geq 30 \%$ & $167(55)$ \\
\hline \multicolumn{2}{|c|}{ The worst mucositis grade during treatment } \\
\hline 0 & $3(1)$ \\
\hline 1 & $16(5)$ \\
\hline 2 & $102(33)$ \\
\hline 3 & $182(60)$ \\
\hline 4 & $2(1)$ \\
\hline \multicolumn{2}{|c|}{ The worst dysphagia grade during treatment } \\
\hline 0 & $12(4)$ \\
\hline 1 & $84(28)$ \\
\hline 2 & $76(25)$ \\
\hline 3 & $133(44)$ \\
\hline 4 & 0 \\
\hline \multicolumn{2}{|c|}{ Resection of primary lesion post-CRT or -BRT } \\
\hline Yes & $30(10)$ \\
\hline No & $275(90)$ \\
\hline \multicolumn{2}{|l|}{ Neck dissection post-CRT or -BRT } \\
\hline Radical neck dissection & 0 \\
\hline Modified radical neck dissection & $1(1)$ \\
\hline Selective neck dissection & $44(14)$ \\
\hline
\end{tabular}

Abbreviations: ECOG Eastern Cooperative Oncology Group, SCC Squamous cell carcinoma, $A C E i$ Angiotensin-converting enzyme inhibitor, ARB Angiotensin II receptor blocker, PPI Proton pump inhibitor, 3D-CRT Three-dimensional conformal radiation therapy, IMRT Intensity-modulated radiation therapy, $C R$ Complete response, CRT Chemoradiotherapy, BRT Bio-radiotherapy The normal range of laboratory data at our institution: Serum albumin (3.8$5.2 \mathrm{~g} / \mathrm{dl}$ ), hemoglobin (male: $13.5-17.6 \mathrm{~g} / \mathrm{dl}$, female: $11.3-15.2 \mathrm{~g} / \mathrm{dl}$ )

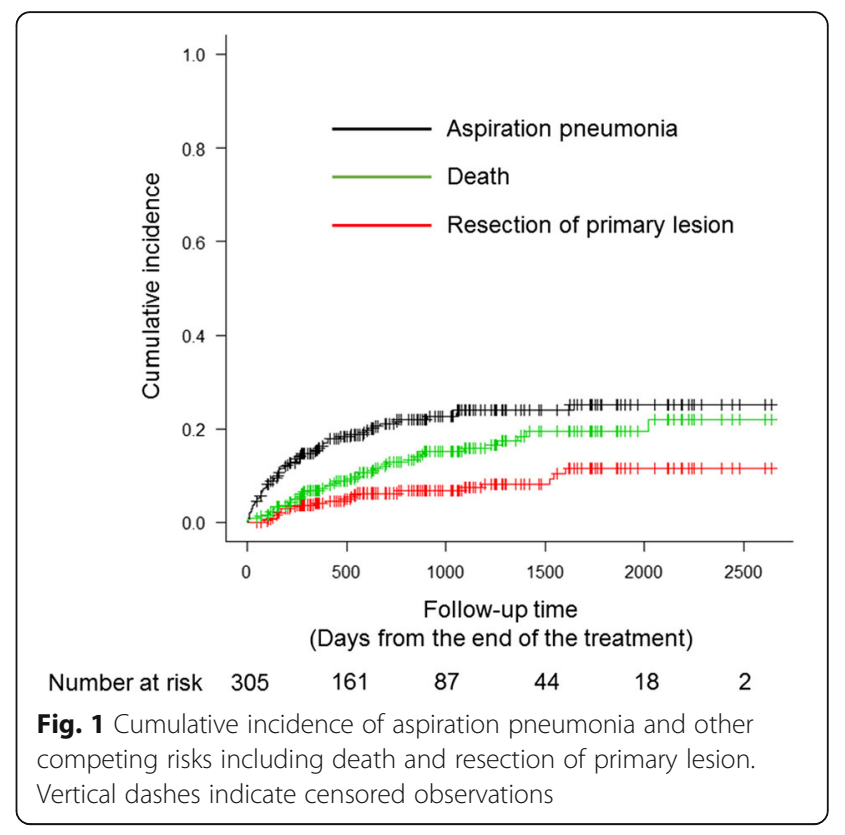

of life post-treatment. Although approximately $60-70 \%$ of patients with HNC treated with CRT suffer from dysphagia or aspiration as a late toxicity $[16,17]$, in previous studies, the incidence of aspiration pneumonia within a year after CRT was found to differ, ranging from 5.4 to $23 \%$ [9, 17, 18]. Furthermore, no differences in the frequency of aspiration pneumonia were seen between the different observation periods, despite the improvement of radiation techniques and general management of CRT over the time. This suggests that various factors other than aspiration are associated with the occurrence of aspiration pneumonia.

To clarify the population at high risk of aspiration pneumonia after CRT or BRT, we investigated the factors predictive of aspiration pneumonia. Several risk factors for aspiration pneumonia in patients with HNC after CRT were reported in previous studies [7, 9, 17]. However, evaluation of the long-term risk factors was often difficult in patients with HNC because these patients' characteristics varied according to the multimodal therapies that they had received, including surgery, CRT, and RT. In particular, previous studies did not take salvage surgery after CRT into account. Therefore, in these studies, there might not have been accurate estimates of the treatment-specific incidence of aspiration pneumonia after CRT. To our knowledge, the current study is the first regarding specific risk factors and predictive models for aspiration pneumonia as a late toxicity in patients with HNC undergoing definitive CRT or BRT.

We intended to determine risk factors for aspiration pneumonia after CRT or BRT by estimating the causespecific cumulative incidence. To do this, we first performed cumulative incidence analysis, and regarded 
Table 2 Univariate and multivariate logistic regression analyses for risk factors of aspiration pneumonia

\begin{tabular}{|c|c|c|c|c|}
\hline & \multicolumn{2}{|l|}{ Univariate analysis } & \multicolumn{2}{|l|}{ Multivariate analysis } \\
\hline & Odds ratio $(95 \% \mathrm{Cl})$ & $P$-value & Odds ratio $(95 \% \mathrm{Cl})$ & $P$-value \\
\hline \multicolumn{5}{|l|}{ Age } \\
\hline$<65$ years old & Ref. & & & \\
\hline$\geq 65$ years old & $1.71(0.97-2.99)$ & 0.06 & & \\
\hline \multicolumn{5}{|l|}{ Gender } \\
\hline Male & $1.06(0.46-2.43)$ & 0.89 & & \\
\hline Female & Ref. & & & \\
\hline \multicolumn{5}{|c|}{ ECOG performance status } \\
\hline $0-1$ & Ref. & & & \\
\hline $2-3$ & $2.61(0.89-7.62)$ & 0.07 & & \\
\hline \multicolumn{5}{|l|}{ Body mass index } \\
\hline$<18.5$ & Ref. & & & \\
\hline $18.5-25$ & $0.87(0.41-1.87)$ & 0.73 & & \\
\hline$\geq 25$ & $0.71(0.27-1.83)$ & 0.48 & & \\
\hline \multicolumn{5}{|l|}{ Primary site } \\
\hline Larynx & Ref. & & & \\
\hline Nasopharynx & $1.21(0.32-4.55)$ & 0.77 & & \\
\hline Hypopharynx & $2.07(0.73-5.83)$ & 0.17 & & \\
\hline Nasal sinus & $1.71(0.36-8.12)$ & 0.49 & & \\
\hline Oropharynx & $3.49(1.23-9.94)$ & 0.02 & $1.69(0.50-5.67)$ & 0.39 \\
\hline Oral cavity & $4.44(1.06-18.7)$ & 0.04 & $1.95(0.38-9.98)$ & 0.42 \\
\hline \multicolumn{5}{|l|}{ T-classification } \\
\hline $1-2$ & Ref. & & & \\
\hline $3-4$ & $2.38(1.32-4.30)$ & 0.004 & $1.75(0.85-3.59)$ & 0.12 \\
\hline \multicolumn{5}{|l|}{ N-classification } \\
\hline $0-2 b$ & Ref. & & & \\
\hline $2 c-3$ & $1.39(0.75-2.57)$ & 0.29 & & \\
\hline \multicolumn{5}{|l|}{ Tumor histology } \\
\hline SCC & Ref. & & & \\
\hline Others & $0.20(0.02-1.57)$ & 0.12 & & \\
\hline \multicolumn{5}{|l|}{ Smoking status } \\
\hline Never & Ref. & & & \\
\hline Past & $0.87(0.37-2.07)$ & 0.76 & & \\
\hline Current & $1.14(0.43-2.98)$ & 0.78 & & \\
\hline \multicolumn{5}{|c|}{ Habitual alcoholic consumption } \\
\hline Yes & $1.79(1.00-3.24)$ & 0.05 & $2.11(1.01-4.38)$ & 0.04 \\
\hline No & Ref. & & & \\
\hline \multicolumn{5}{|c|}{ Distance from the hospital } \\
\hline$<10 \mathrm{~km}$ & Ref. & & & \\
\hline$\geq 10 \mathrm{~km}$ & $0.88(0.48-1.59)$ & 0.67 & & \\
\hline \multicolumn{5}{|c|}{ Family members in the same household } \\
\hline Yes & Ref. & & & \\
\hline No & $1.33(0.64-2.73)$ & 0.44 & & \\
\hline
\end{tabular}


Table 2 Univariate and multivariate logistic regression analyses for risk factors of aspiration pneumonia (Continued)

Use of ACEi or ARB

Yes

No

Use of PPI or $\mathrm{H}_{2}$ blocker

Yes

No

Oral hygiene before treatment

Good

Poor

Coexistence of other malignancies

Yes

No

Comorbidity index

0

$\geq 1$

Serum albumin before treatment

Within normal limits

Less than normal range

Hemoglobin before treatment

Within normal limits

Less than normal range

Use of sleeping pills at the end of treatment

$$
\text { Yes }
$$

No

Main feeding at the end of treatment

Oral

Non-oral

Presence of gastrostomy during the treatment

$$
\text { Yes }
$$

No

Induction chemotherapy

Yes

No

Concurrent chemotherapy regimen

CDDP-based

CBDCA-based

Cetuximab

Radiation technique

Conventional 3D-CRT

IMRT

Irradiation field

Primary alone

Hemi neck
1.50 (0.76-2.93)

Ref.

$1.02(0.58-1.77)$

Ref.

Ref.

$2.63(1.33-5.21)$

0.005

0.007

$2.72(1.30-5.68)$

Ref.

Ref.

$0.59(0.29-1.22)$

Ref.

4.60 (2.37-8.95)

0.000006

$2.70(1.12-6.53)$

0.02

Ref.

$2.62(1.49-4.61)$

0.0008

0.00005

$3.22(1.83-5.67)$

Ref.

Ref.

$1.66(0.93-2.96)$

0.09

$2.6(1.41-4.77)$

Ref.

$1.20(0.64-2.23)$

0.56

Ref.

Ref.

$1.01(0.45-2.25)$

0.98

$1.01(0.38-2.62)$

$1.60(0.76-3.34)$

0.21

Ref.

Ref.

$3.82(0.43-33.5)$
$3.51(1.46-8.42)$

0.005
$2.81(1.28-6.16)$ 
Table 2 Univariate and multivariate logistic regression analyses for risk factors of aspiration pneumonia (Continued)

Whole neck $5.43(0.71-41.6) \quad 0.10$

Treatment efficacy

CR

non-CR

Body weight loss after treatment

$$
\begin{aligned}
& <10 \% \\
& \geq 10 \%
\end{aligned}
$$

Serum albumin decreasing

post-treatment

$$
\begin{aligned}
& <20 \% \\
& \geq 20 \%
\end{aligned}
$$

$$
1.12(0.63-1.97)
$$

Hemoglobin decreasing post-treatment

$$
<30 \%
$$$$
\geq 30 \%
$$

Ref.

The worst mucositis grade during treatment

0-2

$$
3-4
$$

The worst dysphagia grade during treatment

$$
\begin{array}{ll}
0-2 & \text { Ref. } \\
3-4 & 0.59(0.33-1.05)
\end{array}
$$$$
\text { Abbreviations: ECOG Eastern Cooperative Oncology Group, SCC Squ }
$$

Ref.
0.10

0.0009

$1.60(0.81-3.14)$

0.17 blocker, PPI Proton pump inhibitor, 3D-CRT Three-dimensional conformal radiation therapy, IMRT Intensity-modulated radiation therapy, $C R$ Complete response The normal range of laboratory data at our institution: Serum albumin (3.8-5.2 g/dl), hemoglobin (male: 13.5-17.6 g/dl, female: 11.3-15.2 g/dl)

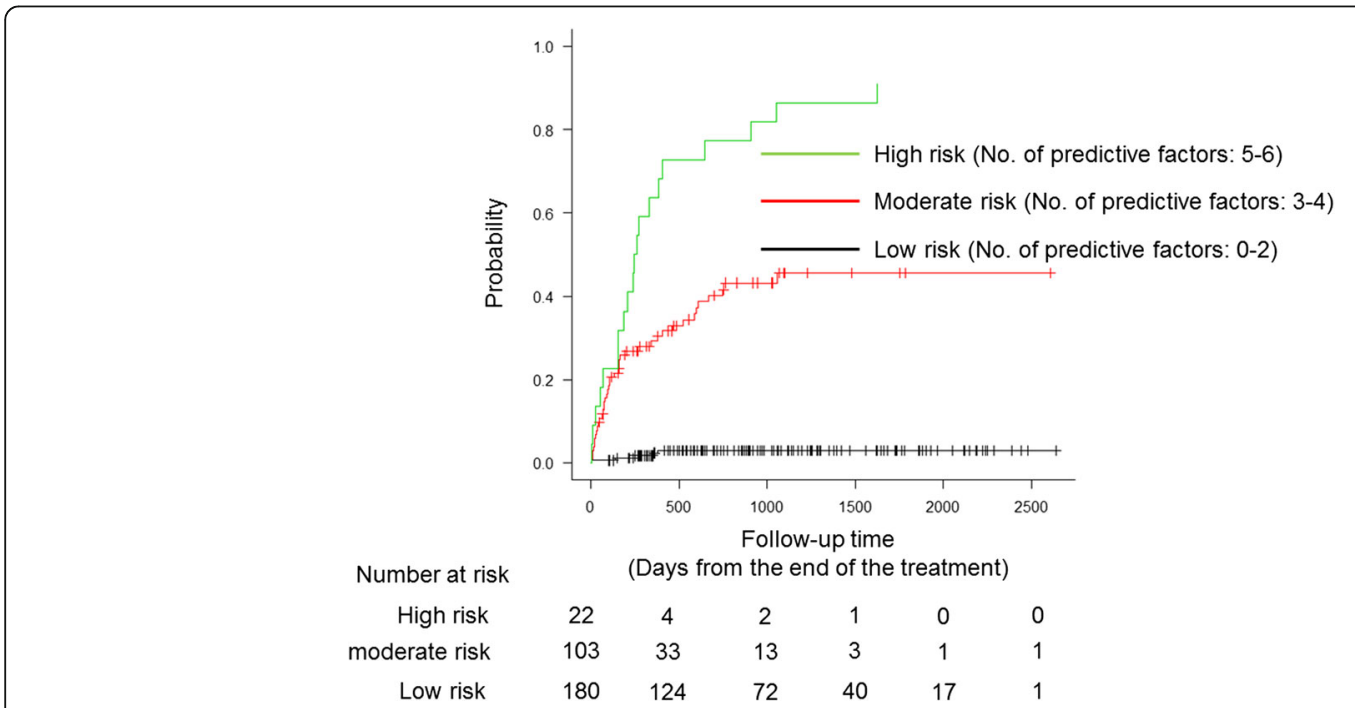

Fig. 2 The estimated cumulative incidence of aspiration pneumonia according to the number of predictive factors. Vertical dashes indicate censored observations 


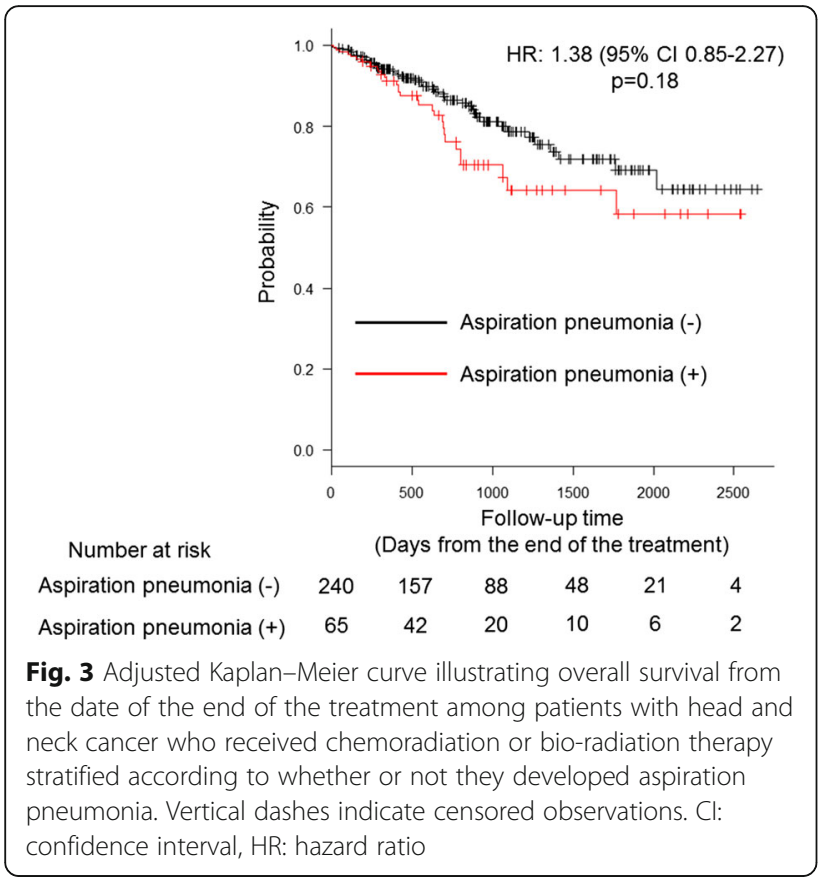

resection of the primary lesion as a competing event. Surgical procedures clearly affect swallowing function. For example, total laryngectomy reduces the risk of aspiration and head and neck reconstruction changes patients' ability to swallow [19, 20]. Therefore, surgical intervention after CRT/BRT may obscure the association of aspiration with CRT or BRT. On the other hand, the effect of neck dissection on aspiration pneumonia has been controversial. For instance, Lango et al. [21]. reported that radical neck dissection (RND) increased the risk of feeding tube dependence in patients with HNC who underwent RT or CRT. On the other hand, Chapuy et al. [22]. reported that types of neck dissection including RND, modified RND, and selective neck dissection (SND) did not aggravate swallowing function. In this study, 45 patients underwent neck dissection, 44 (97\%) of which underwent SND. Our analysis suggested no significant association between neck dissection and the occurrence of aspiration pneumonia $(P=0.23)$. Therefore, we did not consider neck dissection as a competing event in cumulative incidence analysis.

Consistent with previous reports [23], hypoalbuminemia was again identified as a factor predictive of aspiration pneumonia after CRT and BRT in our study. The novel predictive factors identified here were poor oral hygiene, use of sleeping pills, coexistence of other malignancies, and habitual alcohol consumption.

Several studies have demonstrated that careful oral management could reduce the risk of aspiration pneumonia in elderly people and patients with a history of cerebral infarction $[24,25]$. However, few studies have focused on the correlation between oral hygiene and the risk of aspiration pneumonia in patients with HNC. At our institution, patients with HNC undergoing RT have been routinely referred to dentists and received systematic oral care during the treatment [15]. Indeed, 96.0\% of patients received oral evaluation before treatment in this cohort. However, $35.6 \%$ of patients initially evaluated as having poor oral hygiene were still assessed as having this same status after the treatment. This suggested that continuous oral management is required in high-risk patients, even after treatment.

Previous studies suggested that sleeping pills increased the risk of aspiration pneumonia [26, 27]. Among these, benzodiazepines were especially associated with the induction of aspiration through gamma-amino-butyric acid type A (GABA-A) signaling in the lesser esophageal sphincter, in addition to inhibition of the central nervous system [28]. However, in our study, benzodiazepines did not specifically increase the risk of aspiration pneumonia more than other sleeping pills. Notably, 83 out of 94 (88.3\%) patients who used sleeping pills at the end of the treatment continued to use them even after the treatment. Al-Mamgani et al. [29]. demonstrated that 30.7\% of patients with nasopharyngeal cancer who received RT or CRT had the complaint of insomnia during the treatment; however, approximately half of them recovered after the treatment. These findings suggest that the unnecessary administration of sleeping pills might increase the risk of aspiration pneumonia for our patients.

Our data demonstrated that the coexistence of other malignancies was a risk factor for aspiration pneumonia. Of 11 patients who had multiple primary HNC or cervical esophageal cancers simultaneously treated by CRT with main HNC, 7 (63.6\%) developed aspiration pneumonia. A previous report suggested that enlargement of the irradiation field increased the risk of aspiration pneumonia [30]. Furthermore, 18 patients underwent surgical or endoscopic resection for esophageal and gastric cancer. Of these, six (33.3\%) developed aspiration pneumonia, three of whom developed it within one week post-resection. Therefore, we speculated that postsurgical immunosuppression and anesthesia or sedation before endoscopy might deteriorate swallowing function.

Previous reports indicated that alcohol suppressed the cough reflex, reduced consciousness, and promoted gastro-esophageal reflux [31-33]. Therefore, such complex factors induced by habitual alcohol consumption may be involved in the occurrence of aspiration pneumonia.

Scheld et al. [34]. and Xu et al. [7]. reported that aspiration pneumonia was a significant prognostic factor. Furthermore, Szczesniak et al. [6]. reported that aspiration pneumonia accounted for 19\% of non-cancerrelated deaths of patients with HNC who received CRT. Therefore, we expected that aspiration pneumonia 
would be strongly associated with patient survival. However, our study did not show a statistically significant difference in survival between patients who developed aspiration pneumonia and those who did not, probably because of the relatively small number of deaths within the short follow-up period.

Our study had several limitations. First, it involved a retrospective analysis at a single institution. Second, differential diagnosis between aspiration pneumonia and other types of pneumonia was often difficult because the definitions of aspiration pneumonia varied among previous reports [9-11]. Third, the median follow-up of 2.4 years was shorter than in previous studies $[4,7]$. The ability of our predictive model might change upon a long-term follow-up. For example, because submucosal remodeling and neurological disturbance slowly progress after irradiation [35], irradiation might have a stronger impact on the occurrence of aspiration pneumonia at a later phase.

Further studies are warranted to validate our predictive model because of the retrospective nature of this study. However, the strength of our study is that almost all patients received standard chemotherapeutic regimens containing platinum or cetuximab, with systematic supportive care such as oral care. Therefore, our predictive model may be more useful for identifying patients at high risk for aspiration pneumonia in recent clinical practice than previous evidences. For example, we propose that clinicians consider swallowing exercises for high- or moderate-risk groups to improve their swallowing function and subsequently prevent aspiration pneumonia [35].

\section{Conclusions}

We investigated the cause-specific incidence and identified risk factors for aspiration pneumonia following definitive CRT or BRT for patients with locally advanced HNC. The prediction of aspiration pneumonia may be necessary to preserve the quality of life and extend life expectancy for patients. Long-term follow-up and further prospective studies are needed to validate the usefulness of our predictive model.

\footnotetext{
Abbreviations

3D-CRT: Three-dimensional conformal radiation therapy; ACE: Angiotensinconverting enzyme; AIC: Akaike information criterion; ALB: Serum albumin; ARBs: Angiotensin II receptor blockers; BRT: Bio-radiotherapy; CR: Complete response; CRT: Chemoradiotherapy; ECOG: Eastern Cooperative Oncology Group; GABA-A: Gamma-amino-butyric acid type A; Hb: Hemoglobin; HNC: Head and neck cancer; IMRT: Intensity-modulated radiation therapy; OS: Overall survival; PPIs: Proton pump inhibitors; RND: Radical neck dissection; RT: Radiotherapy; SND: Selective neck dissection
}

\section{Acknowledgements}

Not applicable.

Funding

None.

\section{Availability of data and material}

The data analyzed during the current study are available from the corresponding author on reasonable request.

\section{Authors' contributions}

Conceptualization: SK, Writing an original draft: SK, Project administration: TY, Review and editing: TY, YO, AF, Formal analysis: KM, Supervision: $H Y$, Investigation of data: SK, TY, SH, HO, TO, TO, TY, AT, TT, YY, YK, and All authors read and approved the final manuscript.

\section{Competing interests}

The authors declare that they have no competing interests.

\section{Consent for publication}

Not applicable.

\section{Ethics approval and consent to participate}

This study was approved by the Institutional Review Committee of Shizuoka Cancer Center (Decision number 27-J104-27-1) and met the standards set forth in the Declaration of Helsinki. Authors obtained written informed consent from the participants. If it was difficult to get, authors provided the information of this study and patients' right on the website or in a notice board at the hospital.

\section{Author details}

${ }^{1}$ Division of Gastrointestinal Oncology, Shizuoka Cancer Center, 1007 Shimonagakubo, Nagaizumi, Sunto-gun, Shizuoka 411-8777, Japan. Division of Medical Oncology, Shizuoka Cancer Center, Sunto-gun, Shizuoka, Japan. ${ }^{3}$ Division of Radiation Oncology and Proton Therapy, Shizuoka Cancer Center, Sunto-gun, Shizuoka, Japan. ${ }^{4}$ Division of Head and Neck Surgery, Shizuoka Cancer Center, Sunto-gun, Shizuoka, Japan. ${ }^{5}$ Division of Dental and Oral Surgery, Shizuoka Cancer Center, Sunto-gun, Shizuoka, Japan. ${ }^{6} \mathrm{Clinical}$ Research Center, Shizuoka Cancer Center, Sunto-gun, Shizuoka, Japan.

Received: 26 August 2016 Accepted: 10 January 2017

Published online: 17 January 2017

References

1. Adelstein DJ, Li Y, Adams GL, Wagner Jr H, Kish JA, Ensley JF, et al. An intergroup phase III comparison of standard radiation therapy and two schedules of concurrent chemoradiotherapy in patients with unresectable squamous cell head and neck cancer. J Clin Oncol. 2003;21:92-8.

2. Bonner JA, Harari PM, Giralt J, Azarnia N, Shin DM, Cohen RB, et al. Radiotherapy plus cetuximab for squamous-cell carcinoma of the head and neck. N Engl J Med. 2006;354:567-78.

3. Forastiere AA, Goepfert H, Maor M, Pajak TF, Weber R, Morrison W, et al. Concurrent chemotherapy and radiotherapy for organ preservation in advanced laryngeal cancer. N Engl J Med. 2003;349:2091-8.

4. Forastiere AA, Zhang Q, Weber RS, Maor MH, Goepfert H, Pajak TF, et al. Long-term results of RTOG 91-11: a comparison of three nonsurgical treatment strategies to preserve the larynx in patients with locally advanced larynx cancer. J Clin Oncol. 2013;31:845-52.

5. Russi EG, Corvo R, Merlotti A, Alterio D, Franco P, Pergolizzi S, et al. Swallowing dysfunction in head and neck cancer patients treated by radiotherapy: review and recommendations of the supportive task group of the Italian Association of Radiation Oncology. Cancer Treat Rev. 2012:38:1033-49.

6. Szczesniak MM, Maclean J, Zhang T, Graham PH, Cook IJ. Persistent dysphagia after head and neck radiotherapy: a common and underreported complication with significant effect on non-cancer-related mortality. Clin Oncol. 2014;26:697-703.

7. Xu B, Boero IJ, Hwang L, Le QT, Moiseenko V, Sanghvi PR, et al. Aspiration pneumonia after concurrent chemoradiotherapy for head and neck cancer. Cancer. 2015;121:1303-11.

8. Charlson ME, Pompei P, Ales KL, MacKenzie CR. A new method of classifying prognostic comorbidity in longitudinal studies: development and validation. J Chronic Dis. 1987;40:373-83.

9. Mortensen HR, Jensen K, Grau C. Aspiration pneumonia in patients treated with radiotherapy for head and neck cancer. Acta Oncol. 2013;52:270-6. 
10. Chen SW, Yang SN, Liang JA, Lin FJ. The outcome and prognostic factors in patients with aspiration pneumonia during concurrent chemoradiotherapy for head and neck cancer. Eur J Cancer Care. 2010;19:631-5.

11. Nguyen NP, Smith HJ, Dutta S, Alfieri A, North D, Nguyen PD, et al. Aspiration occurrence during chemoradiation for head and neck cancer. Anticancer Res. 2007;27:1669-72.

12. Akaike H. A new look at the statistical model identification. IEEE T Automat Contr. 1974;19:716-23.

13. Harrell Jr FE, Lee KL, Mark DB. Multivariable prognostic models: issues in developing models, evaluating assumptions and adequacy, and measuring and reducing errors. Stat Med. 1996;15:361-87.

14. Kanda Y. Investigation of the freely available easy-to-use software 'EZR' for medical statistics. Bone Marrow Transplant. 2013;48:452-8.

15. Yokota T, Tachibana H, Konishi T, Yurikusa T, Hamauchi S, Sakai K, et al. Multicenter phase II study of an oral care program for patients with head and neck cancer receiving chemoradiotherapy. Support Care Cancer. 2016:24:3029-36.

16. Langerman A, Maccracken E, Kasza K, Haraf DJ, Vokes EE, Stenson KM. Aspiration in chemoradiated patients with head and neck cancer. Arch Otolaryngol Head Neck Surg. 2007;133:1289-95.

17. Eisbruch A, Lyden T, Bradford CR, Dawson LA, Haxer MJ, Miller AE, et al. Objective assessment of swallowing dysfunction and aspiration after radiation concurrent with chemotherapy for head-and-neck cancer. Int Radiat Oncol Biol Phys. 2002;53:23-8.

18. Hunter KU, Lee OE, Lyden TH, Haxer MJ, Feng FY, Schipper M, et al. Aspiration pneumonia after chemo-intensity-modulated radiation therapy of oropharyngeal carcinoma and its clinical and dysphagia-related predictors. Head Neck. 2014;36:120-5.

19. Rathod S, Livergant J, Klein J, Witterick I, Ringash J. A systematic review of quality of life in head and neck cancer treated with surgery with or without adjuvant treatment. Oral Oncol. 2015;51:888-900.

20. Kao SS, Peters MD, Krishnan SG, Ooi EH. Swallowing outcomes following primary surgical resection and primary free flap reconstruction for oral and oropharyngeal squamous cell carcinomas: A systematic review. Laryngoscope. 2016 [Epub ahead of print].

21. Lango MN, Egleston B, Ende K, Feigenberg S, D'Ambrosio DJ, Cohen RB, et al. Impact of neck dissection on long-term feeding tube dependence in patients with head and neck cancer treated with primary radiation or chemoradiation. Head Neck. 2010;32:341-7.

22. Cl C, Annino DJ, Snavely A, Li Y, Tishler RB, Norris CM, et al. Swallowing function following postchemoradiotherapy neck dissection: review of findings and analysis of contributing factors. Otolaryngol Head Neck Surg. 2011;145:428-34

23. Purkey MT, Levine MS, Prendes B, Norman MF, Mirza N. Predictors of aspiration pneumonia following radiotherapy for head and neck cancer. Ann Otol Rhinol Laryngol. 2009;118:811-6.

24. van der Maarel-Wierink CD, Vanobbergen JN, Bronkhorst EM, Schols JM, de Baat C. Oral health care and aspiration pneumonia in frail older people: a systematic literature review. Gerodontology. 2013;30:3-9.

25. Wagner C, Marchina S, Deveau JA, Frayne C, Sulmonte K, Kumar S. Risk of stroke-associated pneumonia and oral hygiene. Cerebrovasc Dis. 2016;41:35-9.

26. Knol W, van Marum RJ, Jansen PA, Souverein PC, Schobben AF, Egberts AC. Antipsychotic drug use and risk of pneumonia in elderly people. J Am Geriatr Soc. 2008;56:661-6.

27. Obiora E, Hubbard R, Sanders RD, Myles PR. The impact of benzodiazepines on occurrence of pneumonia and mortality from pneumonia: a nested case-control and survival analysis in a population-based cohort. Thorax. 2013;68:163-70

28. Rushnak MJ, Leevy CM. Effect of diazepam on the lower esophageal sphincter. A double-blind controlled study. Am J Gastroenterol. 1980; 73:127-30.

29. Al-Mamgani A, van Rooij P, Tans L, Verduijn GM, Sewnaik A, de Jong RJ B. A prospective evaluation of patient-reported quality-of-life after (chemo) radiation for oropharyngeal cancer: which patients are at risk of significant quality-of-life deterioration? Radiother Oncol. 2013;106:359-63.

30. Langendijk JA, Doornaert P, Rietveld DH, Verdonck-de Leeuw IM, Leemans CR, Slotman BJ. A predictive model for swallowing dysfunction after curative radiotherapy in head and neck cancer. Radiother Oncol. 2009;90:189-95.

31. Berkowitz H, Reichel J, Shim C. The effect of ethanol on the cough reflex. Clin Sci Mol Med. 1973:45:527-31.
32. Lee A, Festic E, Park PK, Raghavendran K, Dabbagh O, Adesanya A, et al. Characteristics and outcomes of patients hospitalized following pulmonary aspiration. Chest. 2014;146:899-907.

33. Matsuki N, Fujita T, Watanabe N, Sugahara A, Watanabe A, Ishida T, et al. Lifestyle factors associated with gastroesophageal reflux disease in the Japanese population. J Gastroenterol. 2013;48:340-9.

34. Scheld WM, Mandell GL. Nosocomial pneumonia: pathogenesis and recent advances in diagnosis and therapy. Rev Infect Dis. 1991;13:S743-51.

35. DI R, Lewin JS, Eisbruch A. Prevention and treatment of dysphagia and aspiration after chemoradiation for head and neck cancer. J Clin Oncol. 2006:24:2636-43.

\section{Submit your next manuscript to BioMed Central and we will help you at every step:}

- We accept pre-submission inquiries

- Our selector tool helps you to find the most relevant journal

- We provide round the clock customer support

- Convenient online submission

- Thorough peer review

- Inclusion in PubMed and all major indexing services

- Maximum visibility for your research

Submit your manuscript at www.biomedcentral.com/submit 